University of Nebraska - Lincoln

DigitalCommons@University of Nebraska - Lincoln

$5-1-2000$

\title{
Hysteresis-loop overskewing in the light of a novel nucleation mode
}

Ralph Skomski

University of Nebraska-Lincoln, rskomski2@unl.edu

J. Ping Liu

University of Nebraska-Lincoln, pliu@uta.edu

David J. Sellmyer

University of Nebraska-Lincoln, dsellmyer@unl.edu

Follow this and additional works at: https://digitalcommons.unl.edu/physicssellmyer

Part of the Physics Commons

Skomski, Ralph; Liu, J. Ping; and Sellmyer, David J., "Hysteresis-loop overskewing in the light of a novel nucleation mode" (2000). David Sellmyer Publications. 61.

https://digitalcommons.unl.edu/physicssellmyer/61

This Article is brought to you for free and open access by the Research Papers in Physics and Astronomy at DigitalCommons@University of Nebraska - Lincoln. It has been accepted for inclusion in David Sellmyer Publications by an authorized administrator of DigitalCommons@University of Nebraska - Lincoln. 


\title{
Hysteresis-loop overskewing in the light of a novel nucleation mode
}

\author{
R. Skomski, J. P. Liu, and D. J. Sellmyer \\ Department of Physics and Astronomy and Center for Materials Research and Analysis, \\ University of Nebraska, Lincoln, Nebraska 68588
}

It is demonstrated that the magnetostatic demagnetizing factor is not the only sample-shape dependent contribution to the hysteresis loop. There are also exchange contributions, which lead to interesting effects, particularly in nanostructures. An example is the recently discovered bulging mode in two-phase nanomagnets. The bulging mode exhibits the angular symmetry of the coherent mode, but it is incoherent due to its radial variation and gives rise to negative demagnetizing factors. (C) 2000 American Institute of Physics. [S0021-8979(00)74908-5]

Two-phase nanostructures are of great interest in permanent magnetism and magnetic recording, because the number of pure compounds meeting specific magnetic requirements is limited and the magnetic performance of two-phase structures may be better than that of single-phase magnets. This refers in particular to the permanent-magnet energy product, where an enhancement has been predicted due to the admixture of soft material. ${ }^{1}$ On this basis, room-temperature energy products as high as about $400 \mathrm{~kJ} / \mathrm{m}^{3}$ (50 MGOe) have recently been obtained in iron-rich two-phase $\mathrm{Pt}-\mathrm{Fe}$ thin films. ${ }^{2}$ This energy product is close to energy products of the present record-holder $\mathrm{Nd}_{2} \mathrm{Fe}_{14} \mathrm{~B}$ and clearly exceeds energy products achieved in single-phase PtFe materials.

Since permanent magnets are used in well-defined shapes, ${ }^{3}$ one has to account for the influence of a sample's shape on the hysteretic behavior. A widely used method is hysteresis-loop skewing (shearing), where the magnetization is plotted as a function of the internal field $H^{\prime}=H-D M$ rather than as a function of the applied field $H$. The factor $D$ is usually interpreted as a magnetostatic demagnetizing factor; for homogeneously magnetized ellipsoids of revolution magnetized along the axis of revolution, $D=D_{\|}$equals 0 for long cylinders (needles), 1/3 for spheres, and 1 for thin films. ${ }^{4}$

Here we present theoretical and experimental evidence which questions the purely magnetostatic demagnetizingfield concept and shows that there are sample-shape dependent exchange contributions.

For simplicity, we will restrict ourselves to a nucleationfield analysis of the problem, that is to the reverse magnetic field $\mathbf{H}=-H_{N} \mathbf{e}_{z}$ at which the remanence energy minimum becomes unstable. ${ }^{3,5}$ In the simplest case, nucleation occurs at $\mathbf{M}=\mathbf{M}_{s} \mathbf{e}_{z}$ and leads to complete magnetic reversal, corresponding to a rectangular hysteresis loop whose coercivity $H_{c}$ is equal to $H_{N}$.

A semiphenomenological way of discussing magnetic reversal is Kronmüller analysis. ${ }^{6}$ In the present context we can write

$$
H_{N}=\frac{2 K_{1}}{\mu_{0} M_{s}}-D_{\mathrm{eff}} M_{s},
$$

where $K_{1}$ is the first uniaxial anisotropy constant, $M_{s}$ is the spontaneous magnetization, and $D_{\text {eff }}$ is an effective demagnetizing factor. In structurally homogeneous ellipsoids of revolution having an easy magnetization axis parallel to the axis of revolution there are two exact eigenmodes of interest. If the ellipsoid's radius $R$ is smaller than a coherence radius $R_{\text {coh }}$, then the dominant exchange interaction yields coherent (uniform) nucleation [Fig. 1(a)], whereas for radii larger than

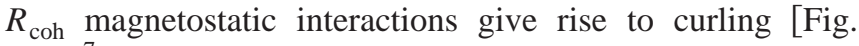
$1(\mathrm{~b})] .^{7}$ For spheres and long cylinders (magnetized needles) one obtains $\quad R_{\text {coh }}=5.099 \sqrt{A / \mu_{0} M_{s}^{2}}$ and $R_{\text {coh }}$ $=3.682 \sqrt{A / \mu_{0} M_{s}^{2}}$, respectively. ${ }^{3,5}$ In these equations, $A$ is the exchange stiffness. As a rule, $R_{\text {coh }}$ is about $10 \mathrm{~nm}$ for a wide range of materials.

Coherent rotation and curling yield the well-known nucleation fields $s^{3,5}$

$$
H_{N}=\frac{2 K_{1}}{\mu_{0} M_{s}}+\frac{1}{2}(1-3 D) M_{s}
$$

and

$$
H_{N}=\frac{2 K_{1}}{\mu_{0} M_{s}}+\frac{c(D) A}{\mu_{0} M_{s} R^{2}}-D M_{s},
$$

respectively. Here $c=8.666$ for spheres $(D=1 / 3)$ and $c$ $=6.780$ for long cylinders $(D=0)$. Comparing Eqs. (2) and (3) with Eq. (1) yields the respective effective demagnetizing factors

$$
D_{\text {eff }}=\frac{1}{2}(3 D-1)
$$

and

$$
D_{\mathrm{eff}}=D-\frac{c(D) A}{\mu_{0} M_{s}^{2} R^{2}} .
$$

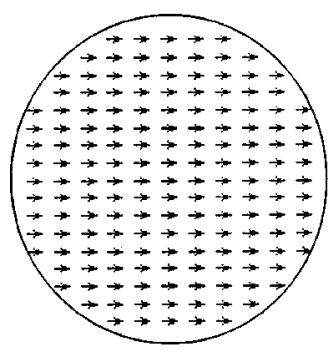

(a)

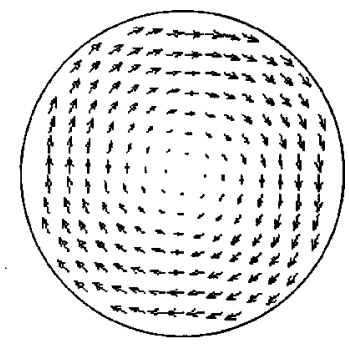

(b)
FIG. 1. Free-surface nucleation modes in a sphere: (a) coherent rotation and (b) curling. The figure shows the $x$ and $y$ magnetization deviations in the $x-y$ plane (top view on the equatorial plane). 
Although these equations are equivalent to Eqs. (2) and (3), they exhibit a number of interesting features: (i) in general, $D_{\text {eff }}$ depends on the radius of the ellipsoid; (ii) the magnetostatic interpretation of the demagnetizing factor $D_{\text {eff }}=D$ is restricted to curling in macroscopic magnets, where $R$ is very large; (iii) interatomic exchange tends to reduce the demagnetizing factor; (iv) the length scale which governs the onset of exchange contributions is the (proper) exchange length $1_{\mathrm{ex}}=\sqrt{A / \mu_{0} M_{s}^{2}}, 8$ and (v) even in the macroscopic limit the result $D_{\text {eff }}=D$ cannot be reduced to a magnetostatic demagnetizing factor. The last comment is related to the fact that the "internal" field acting on the central spin in a spherical magnet is zero rather than $-M / 3$ (see, e.g., p. 58 in Ref. 3). In fact, the appearance of the term $D$ in Eq. (5) has a different origin: from Fig. 1(b) we see that the curling mode exhibits a nonnegligible degree of flux closure, which is energetically favorable and facilitates magnetic reversal. This flux-closure contribution to $D_{\text {eff }}$ cannot be interpreted as a demagnetizing fields in homogeneously demagnetized ellipsoids of revolution, but accidentally it has the same magnitude.

The skewing (shearing) of hysteresis loops is a method to account for the sample-shape dependence of extrinsic magnetic properties, but the experimental aspects of this procedure are by no means trivial. For example, in Ref. 2 the complete neglect of the demagnetizing factor $(D=0)$ gave rise to an unphysically low energy product of about 40 MGOe, whereas putting $D=1$, as appropriate for thin films, would yield an overskewing of the loop with an extrapolated energy product of more than 60 MGOe (Fig. 2). Similar difficulties are encountered in other magnetic systems. By comparing the magnetization curves of $\mathrm{Ni}$ and $\mathrm{Sm}_{2} \mathrm{Fe}_{17} \mathrm{~N}_{3}$ particles fixed in epoxy resin the experimental demagnetizing factors are $D_{\|}=0.14$ and $D_{\perp}=0.33,{ }^{9}$ so that $D_{\|}+2 D_{\perp}$ $=0.80$ rather than $D_{\|}+2 D_{\perp}=1$.

It is instructive to compare our qualitative approach with experimental procedures to circumvent the problem of overskewing. To obtain a reasonable $(B H)_{\max }$ value, Liu et al. ${ }^{2}$ used an approximate skewing procedure based on the assumption of an infinite slope $d M / d H=\infty$ at $H=H_{c},{ }^{10}$ and obtained $(B H)_{\max }=52.8 \mathrm{MGOe}$ for $D=0.48$. The approximate character of this method is evident from a simple counter-example: an ensemble of independent particles having a very broad distribution of coercivities exhibits $d M / d H>0$ at $H=H_{c}$, independently of the strength of the demagnetizing field.

From an experimental point of view it is difficult to separate magnetostatic and exchange contributions to $D_{\text {eff }}$. Both are quadratic in the spontaneous magnetization $M_{s}$ $=|M|$ and have essentially the same temperature dependence, so that they cannot be distinguished by the temperature-dependent measurement methods ${ }^{6,11}$ usually employed to separate $K_{1}$ contributions from magnetostatic contributions. A possibility to trace exchange and magnetostatic contributions to $D_{\text {eff }}$ is to exploit their opposite sign. ${ }^{11}$

The demagnetizing problem becomes even more complicated in two-phase nanostructures. Consider a semihard or soft ferromagnetic sphere of magnetization $M_{s}$, surrounded by and exchange-coupled to a very hard shell of fixed mag-

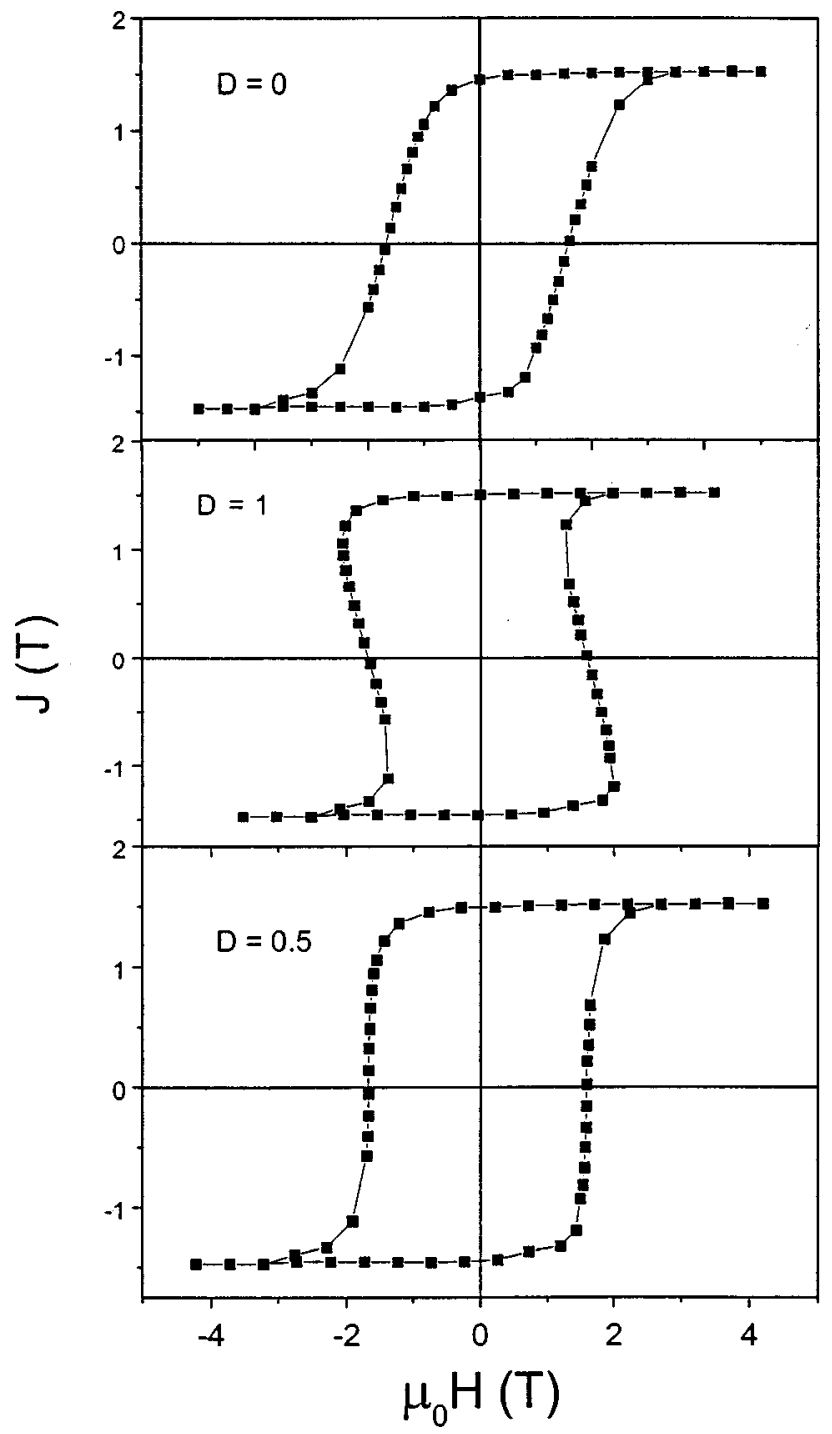

FIG. 2. Demagnetizing-field correction for a Fe/Pt film: (a) raw data, (b) overskewing due to $D=1$, and (c) infinite-slope method $\left(J=\mu_{0} M\right)$. Skewing corrections are of practical importance, because they make it possible to compare the properties of magnets of different shapes.

netization $M_{s} \mathbf{e}_{z}$. This case is not only scientifically interesting but also of practical interest in two-phase nanomagnetism, because soft regions are often embedded in a more or less aligned hard matrix.

The calculation ${ }^{12}$ is similar to the calculation of nucleation modes in homogeneous magnets, but the free-surface boundary condition $\mathbf{n} \cdot \mathbf{M}=0$ must be replaced by clamped boundary conditions $\mathbf{M}=\mathbf{M}_{s} \mathbf{e}_{z}$. It yields two nucleation modes $\mathbf{m}(\mathbf{r})=\mathbf{M}(\mathbf{r})-\mathbf{M}_{s} \mathbf{e}_{z}$ : a curling-type mode characterized by the angular dependence

$$
\mathbf{m}(\mathbf{r})=F(r) \sin (\theta)\left(\cos \phi \mathbf{e}_{y}-\sin \phi \mathbf{e}_{x}\right)
$$

and a purely radial or "quasi-coherent" mode

$$
\mathbf{m}(\mathbf{r})=F(r) \mathbf{e}_{m},
$$




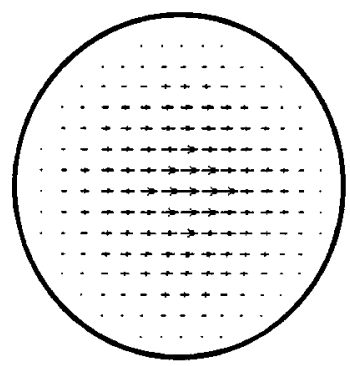

(a)

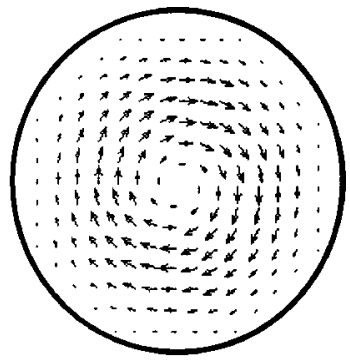

(b)
FIG. 3. Clamped nucleation modes: (a) bulging and (b) modified curling. The figure shows the $x$ and $y$ magnetization deviations in the $x-y$ plane (top view on the equatorial plane).

where $\mathbf{e}_{z} \cdot \mathbf{e}_{m}=0$. The radial eigenfunctions $F(r)$ are spherical Bessel functions subject to clamped boundary conditions. ${ }^{12}$ The quasi-coherent bulging mode [Fig. 3(a)] is characterized by the nucleation field

$$
H_{N}=\frac{2 K_{1}}{\mu_{0} M_{s}}+2 \pi^{2} \frac{A}{\mu_{0} M_{s} R^{2}},
$$

whereas the modified (clamped) curling mode [Fig. 3(b)] exhibits the nucleation field

$$
H_{N}=\frac{2 K_{1}}{\mu_{0} M_{s}}-\frac{1}{3} M_{s}+40.382 \frac{A}{\mu_{0} M_{s} R^{2}} .
$$

The transition between bulging and clamped curling occurs at $R_{\text {coh }}=7.869 \sqrt{A / \mu_{0} M_{s}^{2}}$, which is somewhat larger than for free-surface nucleation. Equation (7) amounts to a negative demagnetizing factor $D_{\text {eff }}=-2 \pi^{2} A / \mu_{0} M_{s}^{2} R^{2}$, whereas Eq. (8) predicts $D_{\text {eff }}<0$ for $R<11.007 \sqrt{A / \mu_{0} M_{s}^{2}}$. ${ }^{13}$

Figure 4 demonstrates the construction of rectangular hysteresis loops from nucleation fields. Essentially, the skewing consists in the replacement of an open-circuit nucleation field $H_{N}$ by the appropriate closed-circuit nucleation field $H_{N^{\prime}}$, but in general this procedure is highly nontrivial. For example, soft inclusions in a hard matrix may interact cooperatively in a curling-type fashion, so that the $D_{\text {eff }}$ exhibits a complicated dependence on the volume fraction of the soft phase.

A related effect is the energy product of very small but prolate (elongated) soft magnets, where interatomic exchange ensures a uniform magnetization state along the axis of revolution and the energy product is $D(1-D) \mu_{0} M_{s}^{2} V .^{3}$ This means that slightly elongated small particles exhibit an energy product of nearly $2 \mu_{0} M_{s}^{2} / 9$, that is $800 \mathrm{~kJ} / \mathrm{m}^{3}[100$ MGOe]. However, due to magnetostatic interparticle interactions, this high energy product cannot be realized in the bulk.

Of course, the model considered ignores that the morphology of real magnets gives rise to for random stray fields and random-anisotropy deviations. This makes the loops

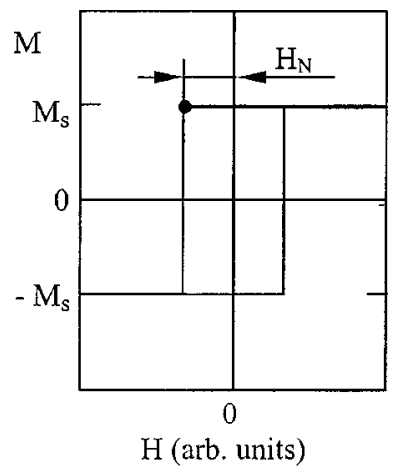

(a)

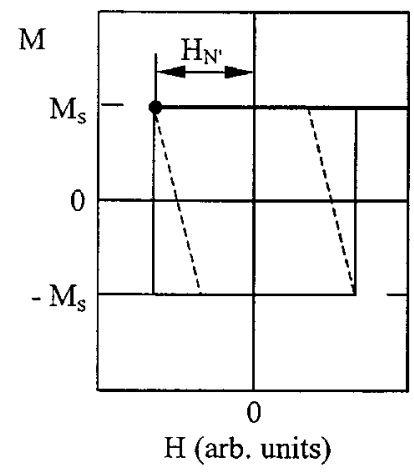

(b)
FIG. 4. Theoretical demagnetizing-field correction: (a) original loop and (b) skewed loop (dashed line). The nucleation fields for the different sample shapes are discussed in the main text.

nonrectangular and modifies the coercivity, but it does not invalidate the qualitative features elaborated in this work.

A more subtle point is the assumption of a "very hard" shell, which leads to the clamped boundary conditions. For very small $R$, where $H_{N}$ is large, the clamped boundary conditions must be replaced by the general boundary conditions introduced in Ref. 1. This indicates that the nucleation mode penetrates from the soft phase into the hard phase (Fig. 2.15 in Ref. 3).

In conclusion, we have shown that the dependence of hysteresis loops on the sample shape cannot be reduced to a homogeneous magnetostatic demagnetizing field. It is, however, possible to define effective demagnetizing factors which, in general, contain a negative exchange contribution. The exchange contribution is particularly important in twophase nanomagnets, where they are associated with reversal mechanisms such as bulging and clamped curling.

This work is supported by NSF and DARPA through ARO DAA655-98-1-0268.

${ }^{1}$ R. Skomski and J. M. D. Coey, Phys. Rev. B 48, 15812 (1993).

${ }^{2}$ J. P. Liu, C. P. Luo, Y. Liu, and D. J. Sellmyer, Appl. Phys. Lett. 72, 483 (1998).

${ }^{3}$ R. Skomski and J. M. D. Coey, Permanent Magnetism (Institute of Physics, Bristol, 1999).

${ }^{4}$ J. A. Osborn, Phys. Rev. 67, 351 (1945)

${ }^{5}$ A. Aharoni, Introduction to the Theory of Ferromagnetism (University Press, Oxford, 1996).

${ }^{6}$ H. Kronmüller, K. D. Durst, and M. Sagawa, J. Magn. Magn. Mater. 74, 291 (1988).

${ }^{7}$ For the buckling mode see, e.g., Ref. 5 .

${ }^{8}$ From relativistic considerations it follows that $l_{\text {ex }}$ scales as $l_{0}=a_{0} / \alpha$ $=7.252 \mathrm{~nm}$, where $a_{0}=0.5292 \AA$ is the Bohr radius and $\alpha=1 / 137$ is Sommerfeld's fine structure constant.

${ }^{9}$ K. Kobayashi, X.-L. Rao, J. M. D. Coey, and D. Givord, J. Appl. Phys. 80, 6385 (1996).

${ }^{10}$ C. D. Mee and E. D. Daniel, Magnetic Recording (McGraw-Hill, New York, 1987), Vol. I, p. 385.

${ }^{11}$ I. A. Al-Omari and D. J. Sellmyer, Phys. Rev. B 52, 3441 (1995).

${ }^{12}$ R. Skomski, J. P. Liu, and D. J. Sellmyer, Phys. Rev. B 60, 7359 (1999).

${ }^{13}$ In a sense, this clamping may be interpreted as a kind of exchange biasing, but this cannot explain the involvement of $A$ in Eq. (3). 